\title{
Convergent evolution of tRNA gene targeting preferences in compact genomes
}

Thomas Spaller ${ }^{1}$, Eva Kling ${ }^{1}$, Gernot Glöckner ${ }^{2,3}$, Falk Hillmann ${ }^{4}$ and Thomas Winckler ${ }^{1 *}$

\begin{abstract}
Background: In gene-dense genomes, mobile elements are confronted with highly selective pressure to amplify without causing excessive damage to the host. The targeting of tRNA genes as potentially safe integration sites has been developed by retrotransposons in various organisms such as the social amoeba Dictyostelium discoideum and the yeast Saccharomyces cerevisiae. In D. discoideum, tRNA gene-targeting retrotransposons have expanded to approximately $3 \%$ of the genome. Recently obtained genome sequences of species representing the evolutionary history of social amoebae enabled us to determine whether the targeting of tRNA genes is a generally successful strategy for mobile elements to colonize compact genomes.

Results: During the evolution of dictyostelids, different retrotransposon types independently developed the targeting of tRNA genes at least six times. DGLT-A elements are long terminal repeat (LTR) retrotransposons that display integration preferences $\sim 15$ bp upstream of tRNA gene-coding regions reminiscent of the yeast Ty3 element. Skipper elements are chromoviruses that have developed two subgroups: one has canonical chromo domains that may favor integration in centromeric regions, whereas the other has diverged chromo domains and is found 100 bp downstream of tRNA genes. The integration of $D$. discoideum non-LTR retrotransposons $\sim 50$ bp upstream (TRE5 elements) and $\sim 100$ bp downstream (TRE3 elements) of tRNA genes, respectively, likely emerged at the root of dictyostelid evolution. We identified two novel non-LTR retrotransposons unrelated to TREs: one with a TRE5-like integration behavior and the other with preference $\sim 4$ bp upstream of tRNA genes.

Conclusions: Dictyostelid retrotransposons demonstrate convergent evolution of tRNA gene targeting as a probable means to colonize the compact genomes of their hosts without being excessively mutagenic. However, high copy numbers of tRNA gene-associated retrotransposons, such as those observed in D. discoideum, are an exception, suggesting that the targeting of tRNA genes does not necessarily favor the amplification of position-specific integrating elements to high copy numbers under the repressive conditions that prevail in most host cells.

Keywords: Dictyostelium, Chromo domain, Chromovirus, Ty3, RNA polymerase III

Abbreviations: APE, Apurinic/apyrimidinic endonuclease; CHD, Chromatin organization modifier domain (chromo domain); CSD, Chromo shadow domain; DGLT-A, Dictyostelium gypsy-like transposable element; DIRS, Dictyostelium intermediate repeat sequence; GAG, Group-specific antigen; IN, Integrase; ITR, Inverted terminal repeat; LTR, Long terminal repeat; mya, Million years ago; ORF, Open reading frame; PBS, Primer binding site; PPy, Polypyrimidine stretch; $\mathrm{RNH}$, Ribonuclease H; RT, Reverse transcriptase; TRE, tRNA gene-associated retroelement
\end{abstract}

\footnotetext{
* Correspondence: t.winckler@uni-jena.de

${ }^{1}$ Institute of Pharmacy, Department of Pharmaceutical Biology, Friedrich

Schiller University Jena, Semmelweisstraße 10, Jena 07743, Germany

Full list of author information is available at the end of the article
}

(c) 2016 The Author(s). Open Access This article is distributed under the terms of the Creative Commons Attribution 4.0 International License (http://creativecommons.org/licenses/by/4.0/), which permits unrestricted use, distribution, and reproduction in any medium, provided you give appropriate credit to the original author(s) and the source, provide a link to the Creative Commons license, and indicate if changes were made. The Creative Commons Public Domain Dedication waiver (http://creativecommons.org/publicdomain/zero/1.0/) applies to the data made available in this article, unless otherwise stated. 


\section{Background}

Mobile elements are obligate genomic parasites that amplify as selfish DNA and play important roles in driving the evolution of their hosts [1-5]. Retrotransposons mobilize by reverse transcription of RNA intermediates and integration of the resulting DNA copies at new locations of their host's genomes. Retrotransposons encode proteins that mediate their mobility and they can be distinguished by their overall structures and retrotransposition mechanisms [6]. The supergroup of retrotransposons bearing long terminal repeats (LTRs) is classified into vertebrate retroviruses (Retroviridae), hepadnaviruses, caulimoviruses, Ty1/copia (Pseudoviridae), Ty3/gypsy (Metaviridae), BEL, and DIRS (Dictyostelium intermediate repeat sequence) [7-9]. NonLTR retrotransposons are a diverse group of mobile elements that lack LTRs and can be further distingushied by structural features such as the presence of an encoded apurinic or apyrimidinic site DNA repair endonuclease or a type IIS restriction endonuclease instead of a retroviral integrase and the presence or absence of a ribonuclease $\mathrm{H}(\mathrm{RNH})$ domain as part of the reverse transcriptase (RT) $[10,11]$.

Dictyostelids are soil-dwelling protists that belong to the supergroup of Amoebozoa [12, 13]. Unfavorable environmental conditions, such as a lack of food, triggers social behaviors in single cells that aggregate and form fruiting bodies to spread some of the population as dormant spores into the environment $[14,15]$. Dictyostelium discoideum, the model organism in studying the biology of social amoebae, has a 34-Mb haploid genome in which two thirds of the chromosomal DNA code for proteins and intergenic regions are mostly below $1 \mathrm{~kb}$ in length [16]. The gene density of this genome limits the available space for transposable elements to expand without causing damage to the host. Therefore, it is remarkable that the genome of $D$. discoideum is interspersed with a variety of mobile elements that add up to nearly $10 \%$ of nuclear DNA [17].

The $D$. discoideum DIRS-1 element has inverted terminal repeats instead of LTRs and a complex arrangement of open reading frames (ORFs) that include an $\mathrm{RT} / \mathrm{RNH}$ and a tyrosine recombinase (YR) instead of a canonical integrase (IN) $[18,19]$ (Fig. 1). DIRS-1 has a strong preference to integrate into existing DIRS-1 copies by a mechanism that probably involves YR-mediated homologous recombination [20]. Therefore, DIRS-1 forms complex clusters located near chromosome ends and contributes $\sim 50 \%$ of centromeric DNA of $D$. discoideum chromosomes [21].

DGLT-A and Skipper are related Ty3/gypsy-type LTR retrotransposons with strikingly different integration preferences. Skipper contains two ORFs coding for enzymatic activities required for retrotransposition arranged in the order RT-RNH-IN [22] (Fig. 1). Skipper

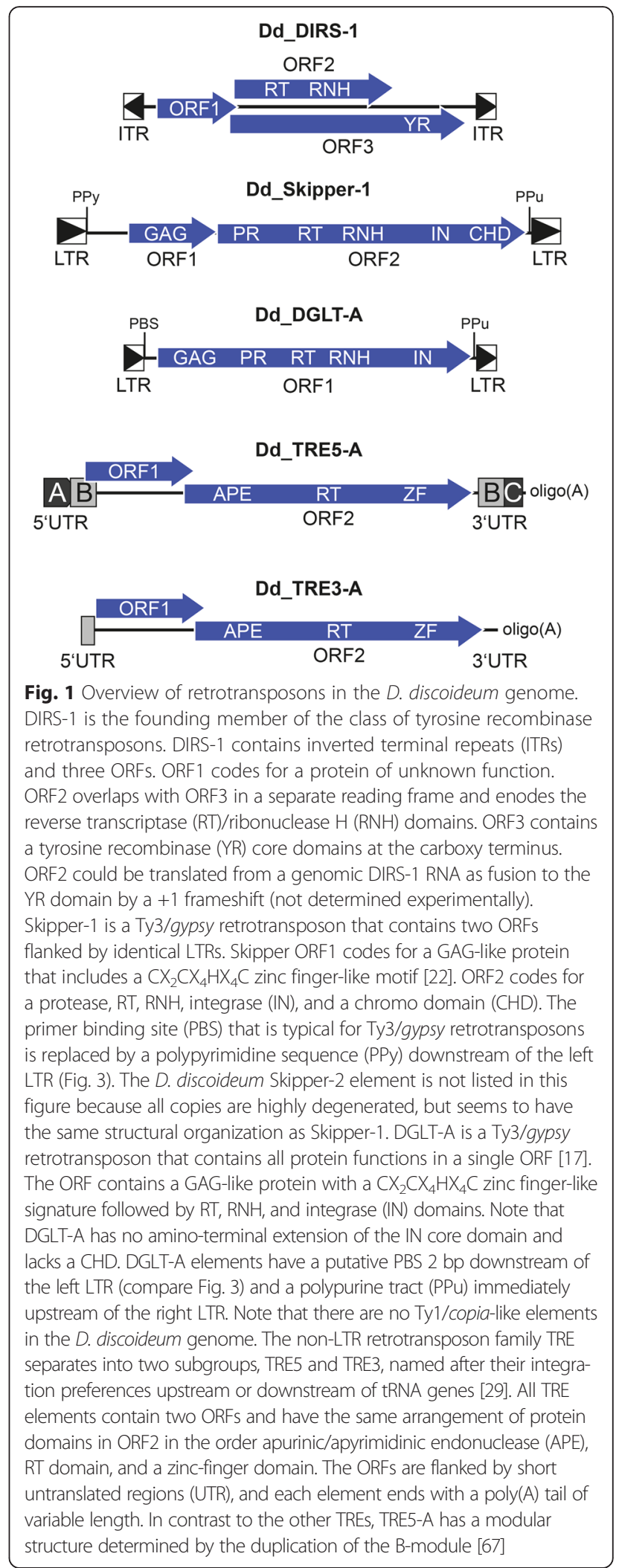


is the prototype chromovirus in the $D$. discoideum genome as it contains a chromo domain (CHD) in the carboxy-terminal extension of the IN protein. The CHD may be responsible for targeting the element to centromeric regions where it contributes to $\sim 10 \%$ of centromer length [21]. It is known that centromeric DNA in $D$. discoideum has properties of heterochromatin including the presence of H3K9 methylation [23]. Retrotransposon CHDs may bind to methylated $\mathrm{H} 3 \mathrm{~K} 9$ and mediate their accumulation in heterochromatin [24], but it has not yet been determined experimentally whether Skipper is tethered to centromers via binding of its $\mathrm{CHD}$ to H3K9 methylation marks.

D. discoideum DGLT-A contains a single ORF and lacks a carboxy-terminal extension of the IN including a CHD as found in Skipper (Fig. 1). DGLT-A is related to Skipper but shows a completely different genomic distribution [17]; it does not accumulate in centromeric DNA but displays a strong preference to integrate within a window of 13-33 bp upstream of the mature coding sequences of tRNA genes [17]. The average distance of DGLT-A to the first nucleotide of a tRNA gene is $15 \mathrm{bp}$. This is remarkably similar to the integration preference of the yeast Ty3 element, considering that Ty3 inserts 1-4 bp upstream of the transcription start sites of tRNA genes [25], which is $\sim 12$ bp upstream of the first nucleotide of mature tRNAs [26]. It is not known whether the molecular mechanism of tRNA gene recognition of DGLT-A resembles that of Ty3, which identifies integration sites by binding of the IN to tDNA-bound transcription factor TFIIIB [27, 28].

The "tRNA gene targeted retroelements" (TREs) form two subfamilies of non-LTR retrotransposons (Fig. 1) that can be distinguished by phylogenetic analysis of their ORF2 proteins [17] and their integration preferences near tRNA genes [29]. TRE5 elements are strictly associated with regions $\sim 50$ bp upstream of tRNA genes, whereas TRE3 elements are always found $\sim 100 \mathrm{bp}$ downstream of tRNA genes. All TREs contain two ORFs. ORF1 proteins of TREs have no similarity among each other or with proteins of non-LTR retrotransposons such as the mammalian L1, in which the ORF1 protein is involved in binding the retroelement's RNA as part of the pre-integration complex and contributes to the integration process $[30,31]$. In $D$. discoideum, the ORF1 protein may be involved in the recognition of tRNA genes as integration sites by binding to subunits of RNA polymerase III transcription factor TFIIIB [32]. The TRE-encoded ORF2 proteins contain related apurinic/ apyrimidinic endonuclease (APE) and RT domains (Fig. 1) that mediate retrotransposition.

It was of interest to trace the evolution of tRNA geneassociated mobile elements in social amoebae to understand how different tRNA gene-directed integration preferences emerged. In this study, we analyzed the annotated genomes of $D$. discoideum, $D$. purpureum, $D$. lacteum, D. fasciculatum, and P. pallidum, which represent the entire evolutionary history of social amoebae $[16,33,34]$. We found that the targeting of tRNA genes has independently developed at least six times through different mobile elements in the evolution of dictyostelids.

\section{Results \\ Retrotransposons have excessively expanded in the $D$. discoideum genome}

Hallmarks of the $D$. discoideum genome are the high gene density and the presence of retrotransposons that closely associate with tRNA genes, likely as a means to avoid insertional mutagenesis of host genes upon retrotransposition. This characteristic of the $D$. discoideum genome is similar to the yeast Saccharomyces cerevisiae, which has an even higher gene density than $D$. discoideum [35] and accommodates only retrotransposons that feature position-specific integration either near tRNA genes or in heterochromatin [36]. It has been of interest to compare integration preferences in yeast and dictyostelid genomes to evaluate whether tRNA gene-targeted integration presents an example of convergent evolution that enables mobile elements to settle in intergenic regions of compact genomes.

We evaluated retrotransposon families in the annotated genomes of $D$. purpureum, D. lacteum, P. palli$d u m$, and $D$. fasciculatum in comparison with the model organism $D$. discoideum. The last common ancestor of all dictyostelids is estimated to date back approximately 600 million years and all examined species featured a long period of separate evolution [33] (Fig. 2), which must be considered when interpreting the relationships among transposable elements both within and outside the dictyostelids. We determined the retrotransposon contents of dictyostelid genomes by performing TBLASTX searches based on $D$. discoideum retrotransposon sequences of the tyrosine recombinase retrotransposon DIRS-1, the LTR retrotransposons Skipper and DGLT-A, and the non-LTR retrotransposons TRE5-A and TRE3-A (the structures of these elements are summarized in Fig. 1). The identified elements were reconstructed as consensus sequences. We also determined whether any of the identified retrotransposons may have a preference for integrating near tRNA genes by searching for tRNA genes within a distance of up to $3000 \mathrm{bp}$ upstream and downstream of identified retroelements. A retrotransposon was considered to display active targeting to tRNA genes if several copies were found in a similar distance to tRNA genes. To ensure that we did not miss tRNA gene-targeting retrotransposons in this analysis, we performed a parallel search in which we first listed all tRNA genes of a given genome and then 


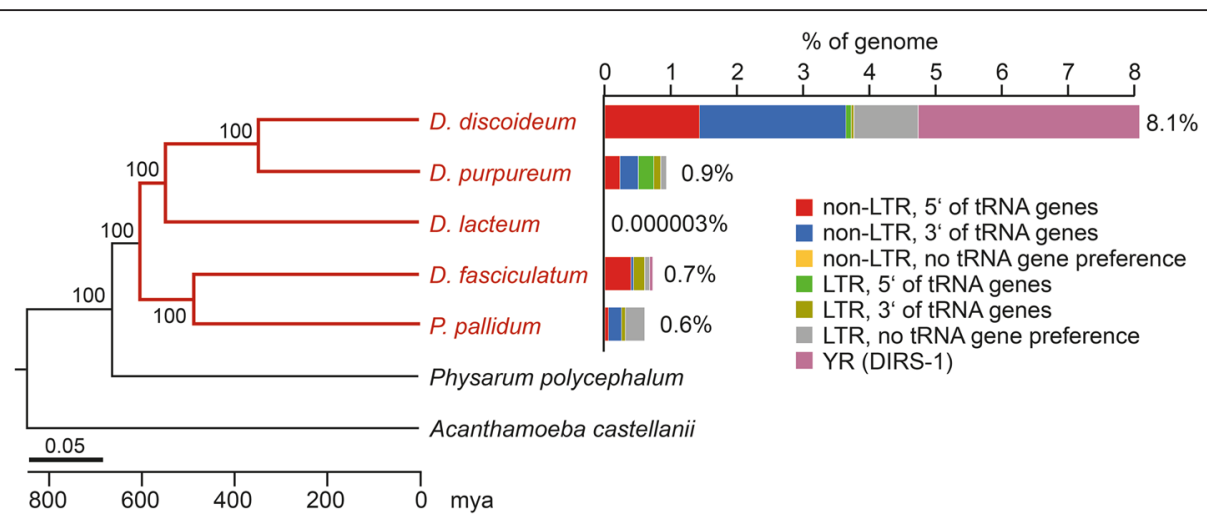

Fig. 2 Phylogenetic relationships between dictyostelids. A genome-based phylogenetic tree was constructed on concatenated sequences of 32 orthologous proteins (redrawn from [13]). The retrotransposon content in each dictyostelid genome is plotted separated by the class of retrotransposon and integration preference near tRNA genes. YR: tyrosine recombinase retrotransposon (DIRS-1)

inspected 3000 bp upstream and downstream sequences for the presence of repetitive elements.

With the exception of $D$. lacteum, which has a particularly small and compact genome, all analyzed dictyostelids have comparable genome sizes of $\sim 30 \mathrm{Mb}$ and gene densities of close to 400 genes/Mb of genomic DNA (Additional file 1: Table S1). A notable difference between the genome of $D$. discoideum and any other examined species is the total retrotransposon content (Fig. 2, Additional file 1: Table S1). Whereas retrotransposons have expanded to $8 \%$ of the $D$. discoideum genome, they have been kept below $1 \%$ in other species.

DIRS-1 has strongly amplified in D. discoideum and constitutes $3.3 \%$ of the genome in this organism [17]. The expansion of Skipper to $1.0 \%$ of the D. discoideum genome may be linked to the amplification of DIRS-1, because both elements reside in centromeric DNA and may have adopted centromer function in this species [21]. Centromeric accumulation of DIRS-1 or Skipper is not observed in any other dictyostelid species except $D$. fasciculatum, which may form small centromeric DIRS clusters that contribute to only $0.1 \%$ of genome size [33]. DIRS-1 is even missing in the assembled sequences of $P$. pallidum and D. purpureum. The data suggest that a putative centromere function of DIRS-1 (and Skipper) as observed in $D$. discoideum is deeply rooted in the social amoebae, even though the majority of species may have evolved deviant strategies to organize their centromeres without allowing the accumulation of selfish mobile elements in these regions.

A notable trend to increase the number of tRNA genes is observed in $D$. discoideum and D. purpureum relative to other dictyostelids (Additional file 1: Table S1). This observation is of interest considering that it may be easier for tRNA gene-targeting retrotransposons to expand if more potential safe integration sites are available. Whereas the tRNA gene-targeting DGLT-A-like retrotransposons are present in low copy numbers in all dictyostelds, a particularly strong amplification in $D$. discoideum relative to other species is observed in the TRE family (Fig. 2, Additional file 1: Table S1). Such expansion is not observed in the genome of $D$. purpureum, which has a comparable amount of tRNA genes. Thus, targeting preference near tRNA genes does not necessarily favor the amplification of position-specific integrating elements to high copy numbers under the repressive conditions that prevail in most host cells.

\section{Dictyostelid LTR retrotransposons comprise related families with different tRNA gene-targeting strategies}

As previously noted by Malik et al. [7], IN domains of Ty3/gypsy-type retrotransposons frequently contain carboxy-terminal extensions including a distinctive GPY/F motif at the end of the IN core followed by relatively unconserved domains of various sizes that may harbor a chromo domain (CHD). D. discoideum DGLTA has a small IN extension of 32 amino acids, whereas Skipper has a long IN extension of 183 amino acids that contains a CHD. In the analysis of dictyostelid genomes described below, we found that all new identified LTR retrotransposons have the Ty3/gypsy-type structure including a conserved GPY/F motif (Additional file 1: Figure S1). For convenience, we call retrotransposons "Skipper" if they contain a CHD in the carboxy-terminal extension of the IN domain and "DGLT-A" if a CHD is lacking.

Twenty insertions of DGLT-A are detectable in the $D$. discoideum genome, eleven of which are solo LTRs that were formerly described as "H3R" elements located upstream of tRNA genes [37]. None of the remaining nine DGLT-A sequences are full-length and refer to the derived consensus of this element (Table 1). This suggests that the DGLT-A population may no longer be able to amplify in the $D$. discoideum genome, even though all 
Table 1 Overview of dictyostelid retrotransposon properties and integration preferences

\begin{tabular}{|c|c|c|c|c|c|c|c|c|}
\hline \multirow[t]{2}{*}{ Name } & \multirow{2}{*}{$\begin{array}{l}\text { Consensus } \\
\text { length (bp) }\end{array}$} & \multirow{2}{*}{$\begin{array}{l}\text { LTR } \\
\text { length } \\
\text { (bp) }\end{array}$} & \multicolumn{3}{|c|}{ Copy number in genome ${ }^{a}$} & \multirow{2}{*}{$\begin{array}{l}\text { tRNA } \\
\text { gene- } \\
\text { specific }\end{array}$} & \multicolumn{2}{|c|}{ Distance to tRNA gene (bp) } \\
\hline & & & total & full length ${ }^{b}$ & solo LTR & & $\begin{array}{l}5^{\prime} \text { of tDNA } \\
(\%)^{c}\end{array}$ & $\begin{array}{l}3^{\prime} \text { of tDNA } \\
(\%)^{c}\end{array}$ \\
\hline \multicolumn{9}{|c|}{ LTR retrotransposons } \\
\hline Dd-Skipper-1 & 6998 & 390 & 60 & 2 & 10 & no & - & - \\
\hline Dp_Skipper-1 & 7485 & 388 & 12 & 1 & 6 & no & - & - \\
\hline DI_Skipper-1 & 4763 & 251 & 7 & 2 & 2 & no & - & - \\
\hline Pp_Skipper-1 & 5589 & 226 & 14 & 1 & 10 & yes & - & - \\
\hline Df_Skipper-1 & $5120^{d}$ & n.d. ${ }^{e}$ & 5 & 0 & n.d. ${ }^{e}$ & no & - & - \\
\hline Pf_Skipper-1.1 & 5296 & 259 & 6 & 1 & 0 & no & - & - \\
\hline Pf_Skipper-1.2 & 6983 & 382 & 3 & 0 & 1 & no & - & - \\
\hline Pf_Skipper-1.3 & 7081 & 363 & 7 & 1 & 6 & no & - & - \\
\hline Dd-Skipper- ${ }^{f}$ & 6178 & 208 & 8 & 0 & 0 & no & - & $8-23(4)$ \\
\hline Dp_Skipper-2 & 5676 & 315 & 23 & 3 & 5 & yes & - & 7-133 (5) \\
\hline Pp_Skipper-2 & $3675^{d}$ & n.d. ${ }^{e}$ & 9 & 0 & n.d. ${ }^{e}$ & yes & - & 54-136 (9) \\
\hline Df_Skipper-2 & 5708 & 312 & 12 & 7 & 5 & yes & - & 26-97 (11) \\
\hline Dd_DGLT-A & 5054 & 265 & 20 & 0 & 5 & yes & 13-33 (18) & - \\
\hline Dp_DGLT-A.1 & 5436 & 492 & 15 & 1 & 9 & yes & $13-16(6)$ & - \\
\hline Dp_DGLT-A.2 & 6114 & 389 & 9 & 2 & 5 & yes & $15(1)$ & - \\
\hline Dp_DGLT-A.3 & 5589 & 563 & 8 & 1 & 6 & yes & $10-11(2)$ & - \\
\hline Dp_DGLT-A.4 ${ }^{\mathrm{g}}$ & $3447^{d}$ & 206 & 30 & 0 & 20 & yes & $16-34(4)$ & - \\
\hline Dp_DGLT-A.5 ${ }^{\mathrm{g}}$ & $3440^{d}$ & 354 & 32 & 0 & 32 & yes & $10-19(15)$ & - \\
\hline DI_DGLT-A.1 & 4895 & 163 & 10 & 1 & 4 & yes & 63-64 (4) & - \\
\hline DI_DGLT-A.2 & 5112 & 206 & 7 & 1 & 2 & yes & $55-65(2)$ & - \\
\hline Pp_DGLT-A.1 & 7295 & 601 & 23 & 1 & 13 & no & - & - \\
\hline Pp_DGLT-A.2 & 6160 & 393 & 12 & 2 & 6 & no & - & - \\
\hline Pp_DGLT-A.3 & 5942 & 212 & 11 & 2 & 3 & no & - & - \\
\hline Pp_DGLT-A.4 ${ }^{\mathrm{g}}$ & $3650^{d}$ & n.d. ${ }^{e}$ & 10 & 0 & n.d. ${ }^{e}$ & yes & 14-24 (3) & - \\
\hline Pf_DGLT-A & 8367 & 168 & 2 & 1 & 1 & no & - & - \\
\hline \multicolumn{9}{|c|}{ Non-LTR retrotransposons } \\
\hline Dd_TRE3-A & 5229 & - & 67 & 13 & - & yes & - & $14-228(60)$ \\
\hline Dd_TRE3-B & 5279 & - & 43 & 9 & - & yes & - & $34-188(39)$ \\
\hline Dd_TRE3-C & 4734 & - & 29 & 2 & - & yes & - & $14-305(29)$ \\
\hline Dd_TRE3-D & $1559^{d}$ & - & 11 & 0 & - & yes & - & 49-285 (11) \\
\hline Dp_TRE3-A & 5150 & - & 56 & 2 & - & yes & - & 69-161 (18) \\
\hline Dp_TRE3-B & 5210 & - & 9 & 2 & - & yes & - & 98-154 (2) \\
\hline Dp_TRE3-C & $1620^{d}$ & - & 37 & 0 & - & yes & - & $67-450(15)$ \\
\hline DI_TRE3-A & 4386 & - & 17 & 2 & - & yes & - & 23/87 (7) \\
\hline Pp_TRE3-A & 4515 & - & 35 & 4 & - & yes & - & $26-138(10)$ \\
\hline Pp_TRE3-B & 4741 & - & 38 & 2 & - & yes & - & $57-151(11)$ \\
\hline Df_TRE3-A & $1867^{d}$ & - & 14 & 0 & - & yes & - & 29-404 (11) \\
\hline Dd_TRE5-A & 5647 & - & 102 & 5 & - & yes & $37-90(98)$ & - \\
\hline Dd_TRE5-B & 5971 & - & 25 & 1 & - & yes & $34-82(25)$ & - \\
\hline Dd_TRE5-C & $879^{d}$ & - & 18 & 0 & - & yes & 38-95 (18) & - \\
\hline DI_TRE5-A ${ }^{h}$ & 7405 & - & 30 & 1 & - & no & - & - \\
\hline
\end{tabular}


Table 1 Overview of dictyostelid retrotransposon properties and integration preferences (Continued)

\begin{tabular}{|c|c|c|c|c|c|c|c|c|}
\hline Pp_TRE5-A & $1169^{d}$ & - & 21 & 0 & - & yes & $38-74(12)$ & - \\
\hline Df_TRE5-A.1 & $2587^{d}$ & - & 56 & 1 & - & yes & $45-88$ (20) & - \\
\hline Df_TRE5-A.2 & $1275^{d}$ & - & 20 & 0 & - & yes & $31-98$ (18) & - \\
\hline Df_TRE5-A.3 & $2941^{d}$ & - & 7 & 0 & - & yes & $39-67$ (6) & - \\
\hline Df_TRE5-B & $1534^{d}$ & - & 27 & 0 & - & yes & $44-90(9)$ & - \\
\hline Dp_NLTR-A & 7438 & - & 28 & 1 & - & yes & $2-6(16)$ & - \\
\hline Pp_NLTR-B & $5550^{d}$ & - & 3 & 0 & - & yes & 39-64 (3) & - \\
\hline Pp_NLTR-C & $3536^{d}$ & - & 12 & 1 & - & no & - & - \\
\hline
\end{tabular}

${ }^{\mathrm{a}}$ Total copy numbers refer to both full-length and partial sequences

${ }^{\text {b }}$ Full-length copies with intact open reading frames

'Distances are listed only for retrotransposons found in the direct neighborhood of tRNA genes; in cases where other tRNA gene-specific retrotransposons have integrated at the same tRNA gene and therefore upstream of a previously inserted element, distances of the original insertion to the target could not be determined. The number of elements used for determination of target distances are shown in parentheses

${ }^{\mathrm{d}}$ No full-length consensus available

${ }^{\mathrm{e}}$ No LTR sequences detectable

fPrevious name DGLT-B (GenBank AF474004) [17]

${ }^{9}$ No ORFs for phylogenetic analysis; classification as DGLT-A according to integration preference

${ }^{\mathrm{h}}$ Classified as TRE5 by similarity of RT sequence (compare Fig. 5)

ORF domains are transcribed in growing $D$. discoideum cells (T.W., unpublished observation).

The $D$. purpureum genome contains three related DGLT-A elements, of which each retained at least one retrotransposition-competent copy. D. purpureum DGLT-As have the same structure and display the same target preference 13-16 bp upstream of tRNA genes as the prototype DGLT-A of $D$. discoideum (Table 1). Two related full-length DGLT-A elements were detected in the D. lacteum genome. These elements also display integration preference upstream of tRNA genes (Table 1). The $P$. pallidum genome contains four related DGLT-A elements. Of these, Pp_DGLT-A.1, Pp_DGLT-A.2, and Pp_DGLT-A.3 comprise a population of elements with intact open reading frames and probable retrotransposition competence. Unlike other DGLT-As, Pp_DGLT-As contain long carboxy-terminal IN extensions of 264-333 amino acids but no detectable CHDs. The IN extensions in P. pallidum DGLT-A elements are poorly conserved among each other and do not show similarity with other retrotransposons such as dictyostelid Skipper or yeast Ty1 and Ty3 elements. Notably, Pp_DGLT-A.1, Pp_DGLT-A.2, and Pp_DGLT-A.3 do not show a preference to integrate near tRNA genes. However, we detected a partial sequence of a fourth DGLT-A in the $P$. pallidum genome (Pp_DGLT-A.4) that is related to the other P. pallidum DGLT-As by phylogenetic analysis of the intact RT and RNH domains (data not shown) and its preference to integrate 14-25 bp upstream of tRNA genes (Table 1). This suggests that the tRNA gene preference of DGLA-A has also been established in the $P$. pallidum genome but was lost in some DGLT-A lineages. The conclusion from this observation is that tRNA gene targeting by DGLT-As was established in the earliest diverged species of Dictyostelia.
The Skipper-1 element of $D$. discoideum is 34 \% identical with DGLT-A in the RT-RNH-IN core domains but does not display integration specificity at tRNA genes. Instead, the approximately 60 Skipper copies are highly enriched in centromeric transposon clusters [21]. Two Skipper copies can be identified in the $D$. discoideum genome that have intact open reading frames and may be retrotransposition-competent.

The D. purpureum genome contains two related Skipper elements. Dp_Skipper-1 is highly similar to Dd_Skipper-1 and does not show association with tRNA genes. In contrast, Dp_Skipper-2, of which three intact copies exist in the D. purpureum genome, is found within a range of 7-133 bp downstream of tRNA genes (Table 1). This integration preference of an LTR retrotransposon had not been observed before. However, in the course of this study, we re-evaluated the previously described DGLT-P element of $D$. discoideum [17] and detected a CHD in the highly degenerated ORF of this element and surprisingly noticed that 4 of 8 copies of this element are located in a range of 8-23 bp downstream of tRNA genes. We therefore renamed DGLT-P "Dd_Skipper-2". Interestingly, a Skipper-like element with target preference downstream of tRNA genes was also detected in the $D$. fasciculatum genome. The Df_Skipper-2 element was found inserted 26-97 bp downstream of tRNA genes, whereas a related Df_Skipper-1 element does not display target specificity (Table 1 ). The $P$. pallidum genome also contains two related Skipper-like elements, of which the Skipper-2 is found within a window of 54136 bp downstream of tRNA genes. The D. lacteum genome contains one intact copy of a Skipper element (Dl_Skipper-1) that is not associated with a tRNA gene. In summary, it seems that Skipper elements diverged into two subfamilies, of which one (Skipper-2) developed 
a previously unnoticed preference to integrate downstream of tRNA genes. This is interesting because integration preference for the same region was also invented by the unrelated non-LTR retrotransposons of the TRE3 family described later.

Phylogenetic analyses based on alignments of the concatenated RT-RNH-IN core domains of all LTR retrotransposons (Additional file 1: Figure S2) support the division of these elements into DGLT-A and Skipper families but also reveal interesting differences in the evolution of these elements (Fig. 3, Additional file 1: Figure S3). For example, DGLT-A elements from $D$. discoideum, $D$. purpureum, and $D$. lacteum form a robust group of elements that share an integration preference upstream of tRNA genes. However, DGLT-A.1, DGLT-A.2, and DGLT-A.3 of P. pallidum clustered with Skipper elements, which was unexpected because P. pallidum DGLT-A.4 (not included in the phylogenetic analysis shown in Fig. 3) showed the DGLT-Atypical integration preference upstream of tRNA genes. On the other hand, the $P$. pallidum DGLT-As that clustered among Skipper elements have long IN extensions reminiscent of Skipper elements, but they lack a detectable CHD.
The phylogenetic analysis presented in Fig. 3 implies a further separation of Skipper elements into two subfamilies: Skipper-1 without target preference and Skipper-2 that integrate downstream of tRNA genes. Notably, all Skipper elements contain carboxy-terminal extensions of the IN core ranging from 99 to 192 amino acid that include distinctive CHDs. The CHDs of Skipper elements are compared in Fig. 4 with the CHD and chromo shadow domain (CSD) of $D$. discoideum heterochromatin protein 1 (HP1), which is known to bind to heterochromatin via its CHD interacting with methylated lysine-9 of histone H3 (H3K9) while its CSD comprises a dimerization domain [38]. Each Skipper-1 retrotransposon contains a canonical HP1-like CHD that has three conserved aromatic amino acids known to build a "cage" responsible for the binding to methylated H3K9 [39] (Fig. 4). Whether CHDs of Skipper-1 elements indeed bind to methylated histone $\mathrm{H} 3$ lysine 9 marks and tether the elements to centromeric regions has not yet been experimentally tested. Gao et al. [24] analyzed CHDs of various LTR retrotransposons and concluded that they can be grouped into "canonical" CHDs (group I CHDs) and derivatives that lack the first and usually also the third of the aromatic cage residues (group II CHDs).

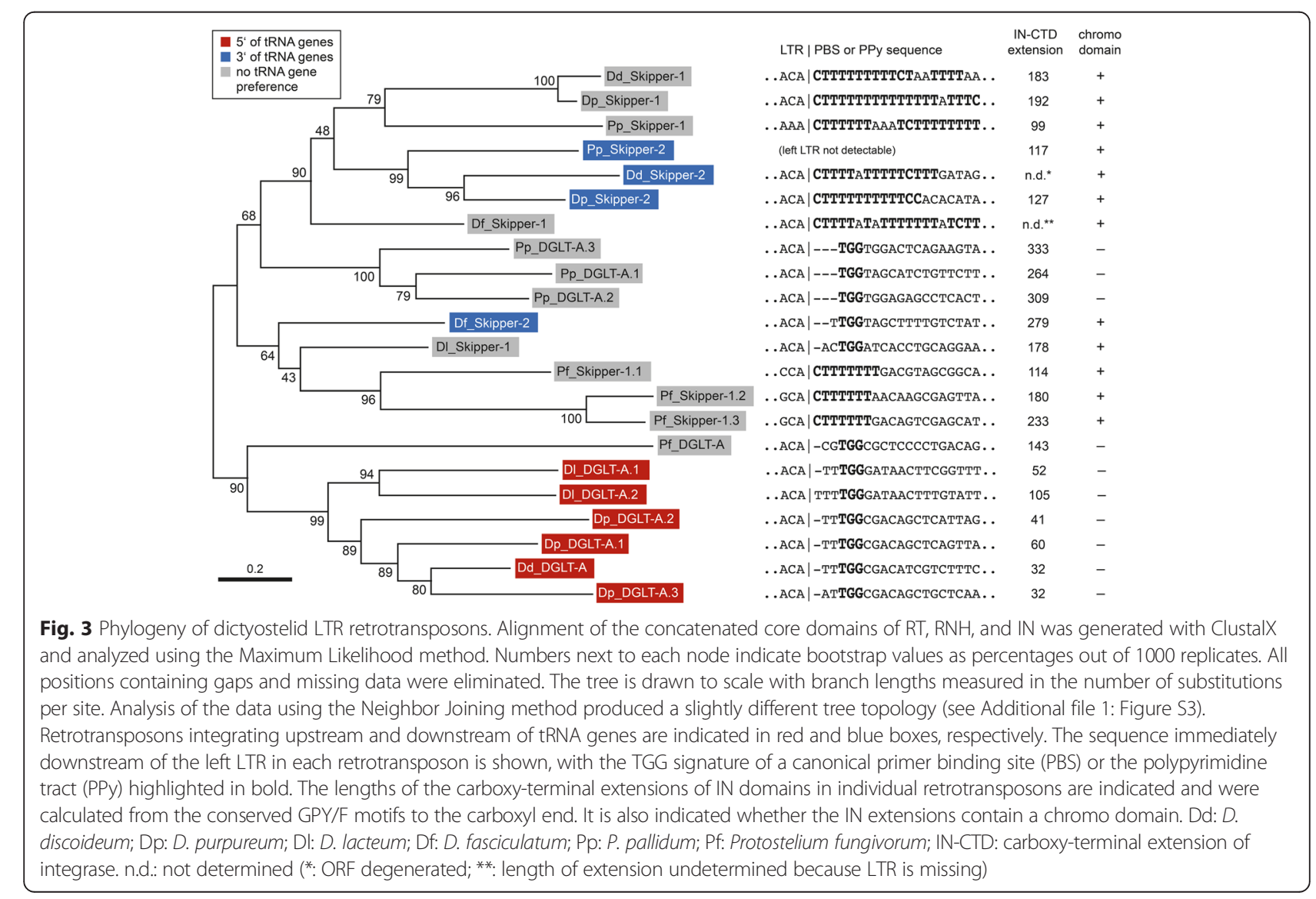




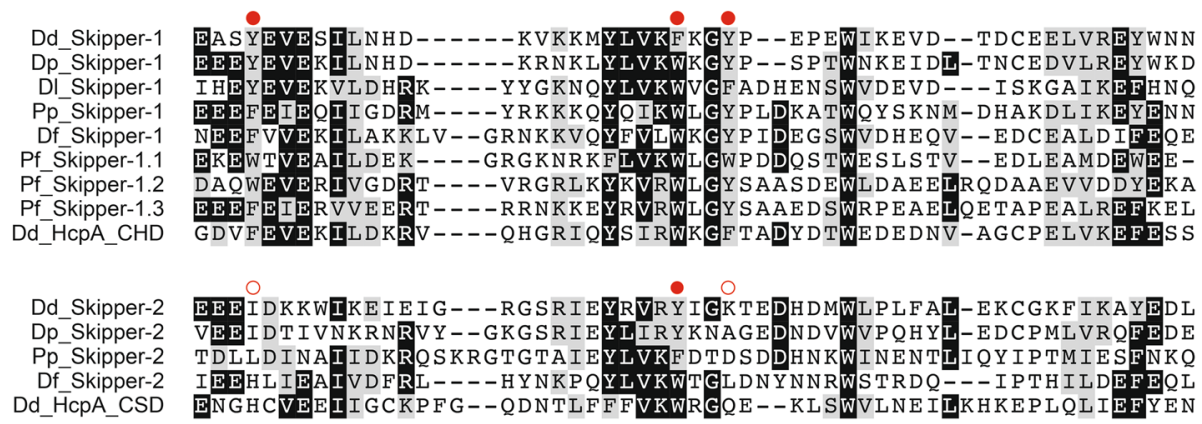

Fig. 4 Alignments of chromo domains in dictyostelid and protostelid Skipper retrotransposons. Alignments were generated with ClustalX. Shading is to a $50 \%$ consensus and was generated with BoxShade. Black boxes indicate invariant amino acids, and gray boxes represent similar amino acids. The corresponding sequences of the chromo domain (CHD) and the "shadow" chromo domain (CSD) of D. discoideum heterochromatin protein 1 (HcpA) [38] are shown for comparison. Red dots depict aromatic cage residues present in canonical chromo domains [39]. The alignment is separated into retrotransposons containing canonical (group I) chromo domains (Skipper-1 elements) and group II chromo domains (Skipper-2 elements) in which the first and third aromatic amino acid of the cage are diverged (indicated by open circles). Dd: Dictyostelium discoideum; Dp: D. purpureum; DI: D. lacteum; Pp: Polysphondylium pallidum; Df: D. fasciculatum; Pf: Protostelium fungivorum

Interestingly, all Skipper-2 elements have diverged exactly the same aromatic cage residues in their CHDs, which in fact resembles the HP1 CSD (Fig. 4). This suggests that CHDs of Skipper-2 elements may be in the process of functional degeneration or, more intriguing, have been modified to shift the integration behavior of these elements to new locations outside of heterochromatin. In this regard, it is of note that Skipper-2 elements apparently evolved a new integration preference downstream of tRNA genes in intergenic regions as described above.

\section{Many Skipper elements have lost the canonical primer binding site to initiate reverse transcription}

A primer binding site (PBS) located immediately downstream of the U5 sequence in the left LTR is required to initiate minus-strand strong-stop cDNA synthesis in most Ty3/gypsy retrotransposons [40, 41]. The PBS usually presents a TGG trinucleotide signature as a complement of the CCA 3' end of a host tRNA that is used as primer to initiate reverse transcription. In $D$. discoideum DGLT-A, the sequence TGGCGACATCGTCTTTC is located $2 \mathrm{bp}$ downstream of the left LTR (Fig. 3), but no tRNA or any other genomic sequence complementary to the PBS could be identified in the $D$. discoideum genome as a potential primer for reverse transcription of DGLT-A.

In contrast to DGLT-A, most elements classified as Skipper according to the presence of a CHD have apparently replaced the canonical PBS with degenerate polypyrimidine (PPy) sequences (Fig. 3) that suggest a noncanonical mechanism of reverse transcription priming. Interesting exceptions are found in Skipper-like elements from D. lacteum and D. fasciculatum: Dl_Skipper-1 has a CHD indicative of Skipper, but contains a PBS typical for DGLT-A. Likewise, Df_Skipper-2 contains a DGLTA-type PBS and a group II CHD. At least seven intact copies Df_Skipper-2 suggest that the element is retrotransposition-competent; all copies are found within a window of 26-97 bp downstream of tRNA genes (Table 1).

\section{The Skipper and DGLT-A families originated before the evolution of dictyostelds}

The long independent evolutionary history of Amoebozoa makes it difficult to trace the origin of DGLT-A- and Skipper-like retrotransposons and the invention of their tRNA gene targeting mechanisms outside the Dictyostelia. The recently obtained genome sequence of a Protostelium species (F.H., T.W., G.G., manuscript in preparation) is helpful, because even though Protostelia are polyphyletic [42], they are considered closer related to the monophyletic Dictyostelia than other amoebozoan species sequenced so far such as Acanthamoeba castellanii or Physarum polycephalum. The genome of the sequenced protostelid, $P$. fungivorum, contains one DGLT-A-like and three Skipper-like elements (Table 1). The Skipper-like elements contain the typical PPy signature downstream of the left LTR (Fig. 3) and a canonical CHD downstream of IN (Fig. 4), supporting the hypothesis that the Skipper-type LTR retrotransposons arose outside the Dictyostelia. Although the gene density of the $P$. fungivorum genome is comparable with the dictyostelids, none of the $P$. fungivorum DGLT-A- or Skipper-like elements has developed integration preferences for tRNA genes. Because the absence of targeting preferences of LTR retrotransposons in this particular Protostelium isolate is not an argument for the de novo invention of such a specificity in dictyostelids, the origin of tRNA gene targeting in dictyostelid genomes remains a mystery until more amoebozoan genomes have been sequenced. 
Dictyostelid non-LTR retrotransposons evolved four different tRNA gene-targeting strategies

In the $D$. discoideum genome, TRE elements can be distinguished between the TRE5 and TRE3 subfamilies according to their exclusive integration behavior [17]. TRE elements comprise $3.7 \%$ of the $D$. discoideum genome, with TRE5-A and TRE3-A contributing the majority of individual copies (Table 1). In D. discoideum, $61 \%$ of tRNA genes are associated with at least one TRE element (Additional file 1: Table S2), and $13 \%$ of tRNA genes have been targeted by both TRE3 and TRE5.

We considered newly discovered non-LTR retrotransposons in dictyostelid genomes as TRE5-like and TRE3-like if they were found upstream and downstream of tRNA genes, respectively, at similar distances as in the $D$. discoideum genome. We examined the evolution of TRE5- and TRE3-like elements using the complete ORF2 sequences of $D$. discoideum TREs as query sequences in TBLASTX searches. We identified TRE5and TRE3-like sequences in D. lacteum, D. fasciculatum and $P$. pallidum, whereas $D$. purpureum contains only TRE3-like sequences (Table 1). Alignments of the conserved RT domains (Additional file 1: Figure S4) and phylogenetic analyses (Fig. 5) support the evolution of TRE5 and TRE3 in separate subfamilies with the exception of Dd_TRE3-C, which appeared to be more related to TRE5 elements than to TRE3 elements in these analyses. This grouping of Dd_TRE3-C is likely caused by the relatively short $\mathrm{RT}$ amino acid sequences used in this analysis because this element clusters robustly with the

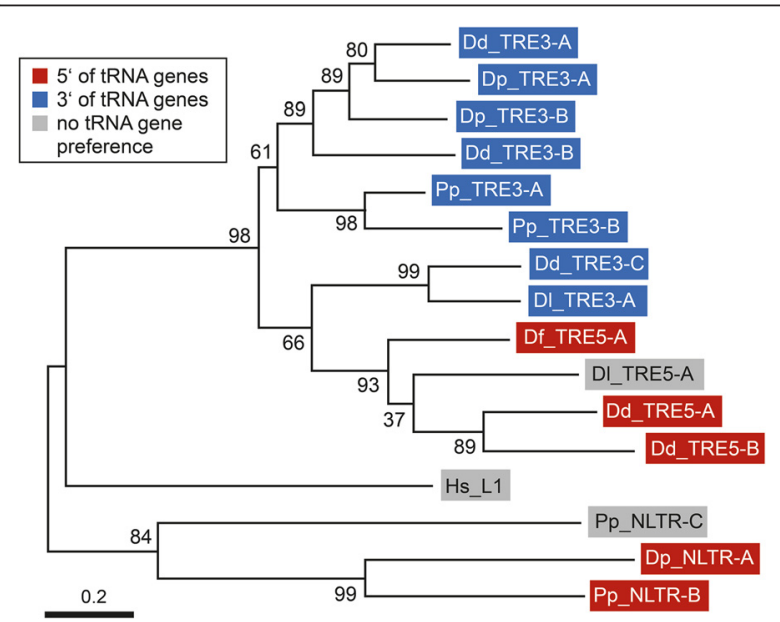

Fig. 5 Phylogenetic analysis of dictyostelid non-LTR retrotransposons. Alignment of RT domains was generated with ClustalX and analyzed using the Maximum Likelihood method. All positions containing gaps and missing data were eliminated. The tree is drawn to scale with branch lengths in the same units as those of the evolutionary distances used to infer the phylogenetic tree. There were a total of 227 amino acid positions in the final dataset. Bootstrap support (percentage from 1000 trials) is indicated next to each node other TRE3 elements when examining the complete ORF2 sequences [17]. Phylogenetic analyses on the entire ORF2 proteins across species was not feasible in this study because complete elements could not be reconstructed in all genomes. TRE-like retrotransposons were found to be associated with tRNA genes at locations typical for $D$. discoideum TRE5 and TRE3 elements (Table 1), suggesting that this type of integration behavior is deeply rooted within the dictyostelids. TRE-like elements have not been identified in the genomes of distantly related amoebozoans such as Physarum polycephalum and Acanthamoeba castellanii and are also absent in the recently sequenced isolate of Protostelium fungivorum. Therefore, the origin of the last common ancestor of the TREs (including the evolution of their unique integration preferences) remains to be determined.

We detected new non-LTR retrotransposons in the genomes of D. purpureum and P. pallidum that we tentatively named "non-LTR" (NLTR) elements because they are only distantly related to TRE elements based on phylogenetic analysis of RT domains (Fig. 5, Additional file 1: Figure S4). D. purpureum NLTR-A and P. pallidum NLTR-B are $38 \%$ identical to each other in their RT domains and are characterized by an RNH domain located downstream of the RT (Fig. 6). Intriguingly, Dp_NLTR-A and Pp_NLTR-B developed different target preferences upstream of tRNA genes (Table 1). Dp_NLTR-A was found 2-6 bp upstream of the first nucleotide of the mature coding sequence of the targeted tRNA gene, which represents an as-yet unobserved integration specificity, whereas Pp_NLTR-B was found at similar positions as TRE5 elements $\sim 50 \mathrm{bp}$ upstream of tRNA genes. $P$. pallidum NLTR-C was identified as a partial sequence that contains an RT domain. This element is only distantly related to Dp_NLTR-A and Pp_NLTR-B ( $26 \%$ sequence identity in the RT domain) and does not show association with tRNA genes. Phylogenetic analysis based on RT domains considering all major subgroups of non-LTR retrotransposons [11] failed to place the Dp_NLTR-A and Pp_NLTR-B elements in any of the subfamilies of non-LTR retrotransposons that are known to harbor an RNH domain (Additional file 1: Figure S5). A phylogenetic evaluation of RNH domains of non-LTR retrotransposons based on alignments previously proposed by Malik et al. [11] confirmed that Dp_NLTR-A and Pp_NLTR-B may form a separate group within the supergroup of non-LTR retrotransposons (Fig. 6; Additional file 1: Figure S6). The Pp_NLTR-C RT sequence aligned best with subgroup R4 elements; however, this grouping could not be evaluated further because no restriction enzyme-like endonuclease domain, which is typically located downstream of RTs in R4-like elements [11], was included in the partial Pp_NLTR-C sequence. 


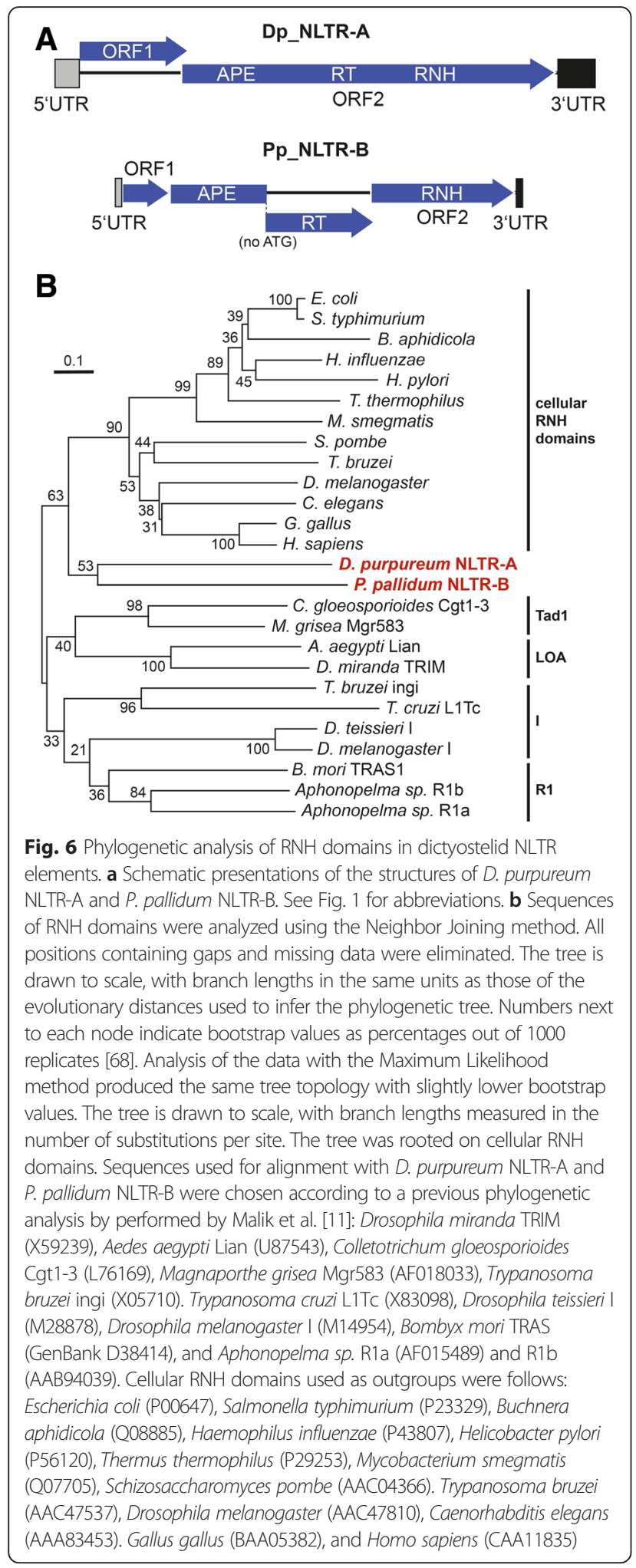

\section{Discussion}

\section{Convergent evolution of integration site selection in compact genomes}

Integration behaviors of retrotransposons residing in compact genomes of different organisms show parallels that suggest strong convergent pressures to avoid insertional mutagenesis of genes and to preserve genome stability of the host. The haploid state of dictyostelid genomes may further increase the selection pressure on mobile elements because the disruption of an essential host gene in the absence of a second compensatory allele would ultimately eliminate the parasite along with its host. In dictyostelids, two principally different strategies have emerged to counter this selection pressure: (i) integration in gene-poor regions of centromeric DNA, which restricts mobile elements to certain spots of repetitive DNA in the host genome and (ii) the targeting of tRNA genes, which not only appears to represent the prime "safe sites" to integrate in gene-rich regions but also enables mobile elements to settle anywhere in the genome due to the multicopy nature of their targets and dispersal on all chromosomes.

In $S$. cerevisiae, the Ty1/copia-type retrotransposon Ty5 is tethered to regions of silent chromatin via direct protein interactions of Ty5 IN with heterochromatinassociated protein Sir4 [43]. There are no Ty1/copiatype retrotransposons found in dictyostelid genomes, but Skipper and DIRS-1 elements accumulate in centromer regions that are organized as heterochromatin. The heterochromatin-targeting mechanisms developed by Skipper and DIRS are different from each other and from Ty5. As we discuss in more detail below, Skipper elements are likely tethered to centromeres via interactions between their chromo domains and histone methylation marks that are characteristic for heterochromatin. The DIRS-1 element is special because it encodes a tyrosine recombinase (YR) instead of a canonical IN and is thought to generate circular retrotransposition intermediates that are probably targeted to centromers via YRmediated homologous recombination into pre-existing DIRS-1 copies $[18,20]$.

The targeting of tRNA genes as presumed safe integration sites has been independently developed at least six times by retrotransposons during dictyostelid evolution (summarized in Fig. 7) and at least twice in the yeast $S$. cerevisiae. Ty1 and Ty3 elements, which belong to different classes of LTR retrotransposons, obviously evolved different mechanisms for tRNA gene recognition. Ty1 integrates within a window of $\sim 750$ bp upstream of tRNA genes that is defined by nucleosome positioning $[44,45]$ and direct interactions between Ty1 IN and RNA polymerase III subunits [46, 47]. A Ty1-like integration behavior of retrotransposons has not been observed in dictyostelid genomes. In contrast, there is a 


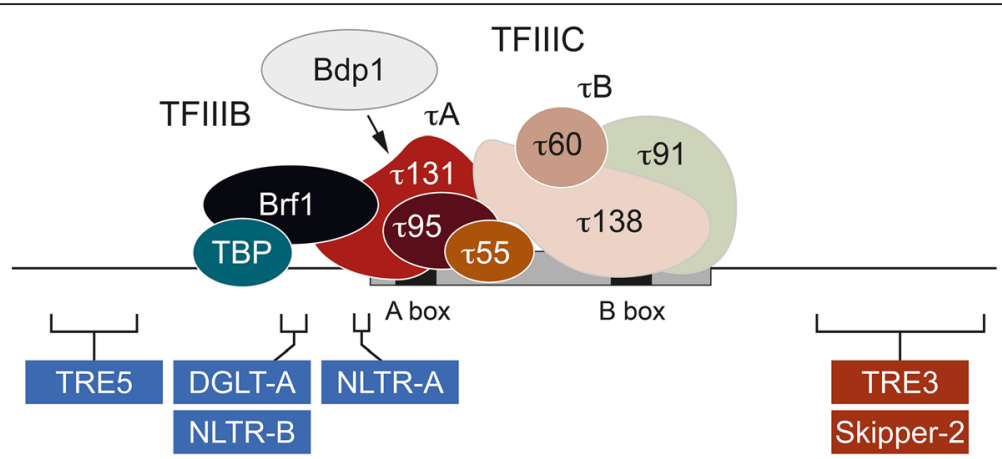

Fig. 7 Summary of integration sites near tRNA genes in dictyostelid genomes. The topology of RNA polymerase III transcription factors TFIIIC and TFIIIB on a tRNA gene is shown as deduced by Male et. al. [69]. The composition of TFIIIB in three subunits is inferred by the presence of orthologs of TBP, Brf1 and Bdp1 in all dictyostelid species. TFIIIC is a six-subunit factor that consists of two subcomplexes, TA and TB [69]. Note that TFIIIB subunit Bdp1 may enter the transcription complex only transiently by displacing TFIIIC subcomplex TB [69]. In dictyostelid genomes only the most conserved TFIIIC subunits T131 (TFC4) and T95 (TFC1) can be identified by homology to either yeast or human orthologs. The schematic is not drawn to scale. The tRNA gene, including its internal regulatory sequences (A box and B box), is indicated as a gray bar. Integration windows in tRNA gene-flanking regions of six different dictyostelid retrotransposon families are indicated. Note that DGLT-A and NLTR-B belong to different retrotransposon classes and therefore independently developed a similar integration behavior upstream of tRNA genes. The same is true for TRE3 and Skipper-2 elements, which target similar regions downstream of tRNA genes

striking similarity of integration site selection between Ty3 and dictyostelid DGLT-A elements. Ty3 targets the entire RNA polymerase III transcriptome of S. cerevisiae [48], particularly in regions $1-4$ bp upstream of the transcription start sites of tRNA genes (that is, $\sim 15 \mathrm{bp}$ upstream of the first nucleotide of the mature tRNA) [25]. This target preference is mediated by an interaction between Ty3 IN and subunits of RNA polymerase III transcription factor TFIIIB [27]. In most dictyostelids evaluated in this study, DGLT-A elements have conserved an integration preference approximately $15 \mathrm{bp}$ upstream of tRNA genes. It would be interesting to determine whether DGLT-A elements use the same molecular interactions to recognize RNA polymerase IIItranscribed genes as $\mathrm{Ty} 3$ or whether selection pressure to avoid gene mutagenesis has generated other solutions to the problem of targeting tRNA gene-upstream regions in different lineages of retrotransposon evolution.

The targeting of tRNA genes by TRE elements is unique to and deeply rooted in the dictyostelids. Although TRE5 and TRE3 elements evolved from a common ancestor [17] that most likely dates back before dictyostelid evolution, these elements developed strikingly different integration preferences and thus use different molecular mechanisms for target recognition. The integration window preferred by TRE3-A elements strikingly overlaps with the integration profile displayed by the unrelated Skipper-2 elements, suggesting that a region $\sim 100$ bp downstream of tRNA genes is accessible for retrotransposons to develop harmless integration strategies in compact genomes. The targeting mechanisms of TRE elements have been investigated experimentally in some detail only in the TRE5-A element, which requires intact $B$ boxes in targeted tRNA genes and probably DNA-bound RNA polymerase III transcription complexes for integration [49]. In vitro data suggest interaction between TRE5-A ORF1 protein and TFIIIB subunits during the integration process [32], which in turn is a remarkable parallel to target recognition by the otherwise unrelated yeast Ty3 element.

Interestingly, high copy numbers of retrotransposons were only found in $D$. discoideum and not in other dictyostelid genomes. Our data suggest that $D$. discoideum is different from the other investigated dictyostelids in that it was specifically affected by an unknown selection pressure that either demanded or coincidentally enabled a burst of retrotransposon expansion. It seems unlikely that the propagation of the sequenced laboratory strain AX4 for about four decades has caused this retrotransposon expansion, because Southern blot data on genomic DNA of the parent strain NC4 probing for TRE5A and TRE3-A indicated similarly high copy numbers of both elements (T.W., unpublished data). It is conceivable that $D$. discoideum has evolved to enable DIRS-1 amplification in centromeres to serve the organism as a substrate for kinetochore complex formation. The tRNA gene-targeting retrotransposons may have profited from this selection and, as a consequence, expanded throughout the genome. However, cells affected in such a manner may have been negatively selected even if there was no direct damage to genes because the haploid genome is particularly vulnerable to non-allelic recombinations forced by the accumulation of repetitive DNA. This consideration may explain why the targeting of tRNA genes by TRE elements achieved a steady state at approximately $60 \%$ saturation of tRNA gene loci. 


\section{Skipper elements may use unconventional priming of reverse transcription}

During the analysis of dictyostelid genomes, the question arose as to whether Skipper elements use a novel mechanism of reverse transcription initiation. Many retroviruses and LTR retrotransposons use cellular tRNAs as primers to initiate minus-strand strong-stop cDNA synthesis [40, 41]. These elements are characterized by a typical TGG trinucleotide signature located a few base pairs downstream of the left LTR that presents the complement of the CCA 3'-end of tRNA primers. All identified DGLT-A elements have this typical TGG motif $2 \mathrm{bp}$ downstream of the left LTR (Fig. 3), but no cellular tRNAs could be identified that may be used as primers for cDNA synthesis. In contrast, most Skipper elements lack the TGG motif and instead contain a degenerate polypyrimidine (PPy) stretch. Although this characteristic feature of Skipper elements could be traced to a Protostelium species suggesting a root outside the dictyostelids, it has not been found in other organisms to the best of our knowledge. Some LTR retrotransposons lacking the TGG signature are assumed to use selfpriming to initiate reverse transcription [50]. In such elements, RNA sequences located in the left LTR at the 5' ends of the retrotransposon transcripts loop back to the region immediately downstream of the LTR and prime reverse transcription [51]. Regarding the Skipper elements, no such complementary regions in the left LTRs are present, suggesting that a novel type of self-priming may be involved. It is unlikely, however, that a "simple" poly(A) stretch somewhere in the Skipper sequence is used in a self-priming process because the PPy sequences in all Skipper elements bear a characteristic $C$ nucleotide facing the orientation of minus-strand cDNA synthesis (Fig. 3).

\section{Dictyostelid Skipper elements are typical chromoviruses}

In D. discoideum, DIRS-1 and Skipper elements form large clusters at the nuclear periphery during interphase that splits into six distinct spots during mitosis representative of the centromeric DNA of the six chromosomes [23]. Interestingly, the clustering of retrotransposons in heterochromatic regions has also been reported in fungal genomes such as that in Magnaporthe grisea, an organism with a similarly high gene density as dictyostelids [52]. This type of retrotransposon clustering appears to differ from the targeting of yeast Ty5 to heterochromatin and likely involves interactions of chromo domains located downstream of IN domains in Ty3/gypsy-type retrotransposons with heterochromatin marks. Similar to DIRS-1, Skipper-1 from D. discoideum has been shown to co-localize with sites of H3K9me2 methylation [23] and binding sites of CenH3, a marker for centromeric heterochromatin [53]. DIRS-1 and Skipper also co-localize with heterochromatin protein 1 (HP1; HcpA), which is recruited to centromeric heterochromatin through the binding of its chromo domain (CHD) to H3K9me2 marks [38]. Skipper shows interesting parallels to centromeric Ty3/gypsy-type retrotransposons bearing CHDs known as chromoviruses, which are found in plants and fungi. For instance, the MAGGY retrotransposon from $M$. grisea targets heterochromatin via interaction with a "canonical" or group I CHD (CHD_I) with histone marks such as H3K9me2 and H3K9me3 [24]. The CHD of Skipper-1 elements is similar to that of D. discoideum HP1 (Fig. 4) and is a representative of group I CHDs (CHD_I); this is consistent with its centromeric accumulation. Some plant chromoviruses contain group II (CHD_II) domains that diverged from CHD_I domains and usually lack the first and third conserved aromatic amino acid that form the "cage" required to interact with methylated histone tails $[24,54]$ (see Fig. 4). CHD_II motifs can tether retrotransposons to heterochromatin without interacting with histone marks [24], yet many CHD_II-bearing chromoviruses are not heterochromatin-associated but spread on chromosomes [55]. CHD_II domains are notably similar to chromo shadow domains (CSD), which are required to mediate the homo- and heterodimerization of HP1 proteins, for instance, in D. discoideum [38]. Thus, CSDs may represent protein interaction platforms that mediate the integration of CHD_II-bearing chromoviruses into heterochromatin by recognizing specific heterochromatinassociated factors [24]. It is tempting to speculate that the divergence of CHD_II domains from canonical CHDs in Skipper-2 elements enabled the development of a new integration preference away from centromeric DNA into intergenic regions downstream of tRNA genes. Interestingly, the transition from CHD_I to CHD_II domains in plant chromoviruses was estimated to date back 500-400 mya [54], which is approximately the time ( 600 mya) when the dictyostelids began to evolve from their last common ancestor [33].

\section{Conclusions}

In the environments of gene-dense genomes, retrotransposons from organisms as divergent as dictyostelid social amoebae and budding yeast reveal convergent evolution leading to the selection of tRNA gene-flanking sequences as potential safe integration sites. In the evolution of dictyostelids, at least six inventions of targeted integration can be discriminated by the choice of distinct integration windows upstream or downstream of tRNA genes by phylogenetically distinctive retrotransposons. In $D$. discoideum, the strong preference of TRE family retrotransposons to integrate near tRNA genes has likely promoted their expansion to almost $4 \%$ of the genome; however, comparing different dictyostelid genomes 
suggests that $D$. discoideum is an exception to the rule and may have been affected by an unknown evolutionary force that either demanded or coincidentally enabled a burst of retrotransposon amplification in this particular species. In general, it is evident from our analysis that non-mutagenic retrotransposition is not a license to amplify possibly because host cells keep track of their repetitive sequences to maintain genome stability.

\section{Methods}

Annotated genome sequences of $D$. discoideum [16], $D$. purpureum [34], D. fasciculatum [33], and P. pallidum [33] were accessed at dictyBase (http://dictybase.org/) [56]. A genome sequence of $D$. lacteum was obtained from Genbank (LODT01000000). The genome sequence of Protostelium fungivorum will be reported elsewhere (F.H., T.W., G.G., manuscript in preparation).

To identify new retrotransposons in dictyostelid genomes, known retrotransposon sequences from $D$. discoideum [17] were used as queries in TBLASTX searches with a cutoff value of $\mathrm{e}<10^{-15}$. Found sequences were expanded by $3000 \mathrm{bp}$ upstream and downstream and analyzed using Jemboss [57]. Blast searches were performed to construct consensus sequences from DNA alignments of individual retrotransposon copies. Searches for LTR sequences were performed using LTR_FINDER [58] to determine full-length LTR retrotransposons and to identify solo LTR sequences. Flanking sequences of retrotransposon copies were analyzed for the presence of tRNA genes using tRNAscan-SE [59] and ARAGORN [60]. To specifically search for tRNA gene-associated retrotransposons, tRNA genes were identified genome-wide using tRNAscan-SE [59] and ARAGORN [60] and listed with $3000 \mathrm{bp}$ upstream and downstream flanking regions for BLASTN searches using the aforementioned identified retrotransposon sequences as queries. Consensus sequences of full-length retrotransposons have been deposited in Repbase (http://www.girinst.org/repbase/) [61]. The following elements have alternative names in Repbase: Pp_Skipper-1: Gypsy-1_PPP; Pp_DGLT-A.1: Gypsy-3_PPP; Pp_DGLT-A.2: Gypsy-2_PPP; Pp_DGLTA.3: Gypsy-4_PPP; Df_Skipper-1: Gypsy-2_DFa; Df_Skipper-2: Gypsy-1_DFa.

For phylogenetic analyses of LTR retrotransposons, the core domains of reverse transcriptase (RT), ribonuclease $\mathrm{H}(\mathrm{RNH})$, and integrase (IN) were determined by searching the Conserved Domain Database [62]. Alignments were generated using ClustalX [63], and conserved amino acid positions were highlighted using BoxShade (http://www.ch.embnet.org/software/BOX_form.html). Shading is to a $50 \%$ consensus with black boxes indicating invariant amino acids and gray boxes representing similar amino acids. Phylogenetic analyses were conducted using the MEGA7 software package
[64]. Phylogenetic trees were analyzed using the NeighborJoining [65] or the Maximum Likelihood method [66] as indicated in the figures.

\section{Additional file}

\begin{abstract}
Additional file 1: Figure S1. Alignments of GPY/F motifs in the carboxy-terminal regions of integrase domains in dictyostelid LTR retrotransposons. Figure S2. Alignments of RT, RNH, and IN domains of dictyostelid LTR retrotransposons. Figure S3. Phylogenetic analysis of dictyostelid LTR elements. Figure S4. Alignment of RT domains of dictyostelid non-LTR retrotransposons. Figure S5. Phylogenetic analysis of RT domains of dictyostelid non-LTR elements. Figure S6. Alignment of dictyostelid non-LTR elements bearing RNH domains. Table S1. Comparison of retrotransposon in annotated dictyostelid genomes. Table S2. Association of tRNA genes with TREs in the D. discoideum genome. (PDF $7243 \mathrm{~kb}$ )
\end{abstract}

\section{Acknowledgments}

This work was supported by grant WI 1142/10-1 from the Deutsche Forschungsgemeinschaft.

\section{Funding}

Grant WI 1142/10-1 (Deutsche Forschungsgemeinschaft) to T.W.

\section{Availability of data and materials}

Consensus sequences of full-length retrotransposons have been deposited in Repbase (http://www.girinst.org/repbase/).

\section{Authors' contributions}

TW conceived the study. TW, TS, EK, FH and GG designed and performed the experiments and revised the manuscript critically for important intellectual content. TW, TS, EK, FH and GG analyzed the data and drafted the manuscript. All authors read and approved the final manuscript.

\section{Competing interests}

The authors declare that they have no competing interests.

\section{Consent for publication}

The manuscript does not contain individuals's personal data.

\section{Ethics approval and consent to participate}

The study does not human participants, human data or tissue and does not include experiments involving animal.

\section{Author details}

${ }^{1}$ Institute of Pharmacy, Department of Pharmaceutical Biology, Friedrich Schiller University Jena, Semmelweisstraße 10, Jena 07743, Germany. ${ }^{2}$ Institute for Biochemistry I, Medical Faculty, University of Cologne, Berlin, Germany. ${ }^{3}$ Institute for Freshwater Ecology and Inland Fisheries, IGB, Berlin, Germany. ${ }^{4} J u n i o r$ Research Group Evolution of Microbial Interaction, Leibniz Institute for Natural Product Research and Infection Biology-Hans Knöll Institute, Jena, Germany.

Received: 23 June 2016 Accepted: 20 August 2016

Published online: 31 August 2016

\section{References}

1. Deininger PL, Moran JV, Batzer MA, Kazazian HH. Mobile elements and mammalian genome evolution. Curr Opin Genet Dev. 2003;13:651-8.

2. Kazazian HH. Mobile elements: drivers of genome evolution. Science. 2004; 303:1626-32.

3. Cordaux R, Batzer MA. The impact of retrotransposons on human genome evolution. Nat Rev Genet. 2009;10:691-703.

4. Levin HL, Moran JV. Dynamic interactions between transposable elements and their hosts. Nat Rev Genet. 2011;12:615-27.

5. Mita P, Boeke JD. How retrotransposons shape genome regulation. Curr Opin Genet Dev. 2016;37:90-100. 
6. Craig NL, Craigie R, Gellert M, Lambowitz AM, editors. Mobile DNA II. Washington DC: ASM Press; 2002

7. Malik HS, Eickbush TH. Modular evolution of the integrase domain in the Ty3/gypsy class of LTR retrotransposons. J Virol. 1999;73: 5186-90.

8. Marin I, Llorens C. Ty3/Gypsy retrotransposons: description of new Arabidopsis thaliana elements and evolutionary perspectives derived from comparative genomic data. Mol Biol Evol. 2000;17:1040-9.

9. Malik HS, Eickbush TH. Phylogenetic analysis of ribonuclease $\mathrm{H}$ domains suggests a late, chimeric origin of LTR retrotransposable elements and retroviruses. Genome Res. 2001;11:1187-97.

10. Malik HS. Ribonuclease $\mathrm{H}$, evolution in retrotransposable elements. Cytogen Genome Res. 2005;110:392-401.

11. Malik HS, Burke WD, Eickbush TH. The age and evolution of non-LTR retrotransposable elements. Mol Biol Evol. 1999:16:793-805.

12. Romeralo M, Skiba A, Gonzalez-Voyer A, Schilde C, Lawal HM, Kedziora S, et al. Analysis of phenotypic evolution in Dictyostelia highlights developmental plasticity as a likely consequence of colonial multicellularity. Proc Biol Sci. 2013;280:20130976.

13. Glöckner G, Noegel AA. Comparative genomics in the Amoebozoa clade. Biol Rev. 2013;88:215-25.

14. Loomis WF. Genetic control of morphogenesis in Dictyostelium. Dev Biol. 2015:402:146-61.

15. Du Q, Kawabe Y, Schilde C, Chen ZH, Schaap P. The evolution of aggregative multicellularity and cell-cell communication in the Dictyostelia. J Mol Biol. 2015;427:3722-33.

16. Eichinger $L$, Pachebat JA, Glöckner G, Rajandream M-A, Sucgang R, Berriman $\mathrm{M}$, et al. The genome of the social amoeba Dictyostelium discoideum. Nature. 2005:435:43-57.

17. Glöckner G, Szafranski K, Winckler T, Dingermann T, Quail M, Cox E, et al. The complex repeats of Dictyostelium discoideum. Genome Res. 2001; 11:585-94.

18. Capello J, Handelsman K, Lodish HF. Sequence of Dictyostelum DIRS-1: An apparent retrotransposon with inverted terminal repeats and an internal circle junction sequence. Cell. 1985;43:105-15.

19. Poulter RTM, Goodwin TJD. DIRS-1 and the other tyrosine recombinase retrotransposons. Cytogenet Genome Res. 2005;110:575-88.

20. Capello J, Cohen SM, Lodish HF. Dictyostelium transposable element DIRSpreferentially inserts into DIRS-1 sequences. Mol Cell Biol. 1984;4:2207-13.

21. Glöckner G, Heidel AJ. Centromere sequence and dynamics in Dictyostelium discoideum. Nucleic Acids Res. 2009;37:1809-16.

22. Leng P, Klatte DH, Schumann G, Boeke JD, Steck TL. Skipper, an LTR retrotransposon of Dictyostelium. Nucleic Acids Res. 1998;26:2008-15.

23. Kaller M, Földesi B, Nellen W. Localization and organization of protein factors involved in chromosome inheritance in Dictyostelium discoideum. Biol Chem. 2007;388:355-65.

24. Gao X, Hou Y, Ebina H, Levin HL, Voytas DF. Chromodomains direct integration of retrotransposons to heterochromatin. Genome Res. 2008;18: 359-69.

25. Chalker DL, Sandmeyer SB. Ty3 integrates within the region of RNA polymerase III transcription initiation. Genes Dev. 1992;6:117-28.

26. Hopper AK. Transfer RNA, post-transcriptional processing, turnover, and subcellular dynamics in the yeast Saccharomyces cerevisiae. Genetics. 2013; 194:43-67.

27. Qi X, Sandmeyer S. In vitro targeting of strand transfer by the Ty3 retroelement integrase. J Biol Chem. 2012;287:18589-95.

28. Yieh L, Kassavetis GA, Geiduschek EP, Sandmeyer SB. The Brf and TATAbinding protein subunits of the RNA polymerase III transcription factor IIIB mediate position-specific Integration of the gypsy-like element, Ty3. J Biol Chem. 2000;275:29800-7.

29. Szafranski K, Glöckner G, Dingermann T, Dannat K, Noegel AA, Eichinger L, et al. Non-LTR retrotransposons with unique integration preferences downstream of Dictyostelium discoideum transfer RNA genes. Mol Gen Genet. 1999:262:772-80.

30. Kolosha VO, Martin SL. High affinity, non-sequence-specific RNA binding by the open reading frame 1 (ORF1) protein from long interspersed nuclear element 1 (LINE-1). J Biol Chem. 2003;278:8112-7.

31. Martin SL, Bushman D, Wang F, Li PW-L, Walker A, Cummiskey J, et al. A single amino acid substitution in ORF1 dramatically decreases L1 retrotransposition and provides insight into nucleic acid chaperone activity. Nucleic Acids Res. 2008;18:5845-54.
32. Chung $\mathrm{T}$, Siol $\mathrm{O}$, Dingermann $\mathrm{T}$, Winckler $\mathrm{T}$. Protein interactions involved in tRNA gene-specific integration of Dictyostelium discoideum non-long terminal repeat retrotransposon TRE5-A. Mol Cell Biol. 2007;27:8492-501.

33. Heidel AJ, Lawal HM, Felder M, Schilde C, Helps NR, Tunggal B, et al. Phylogeny-wide analysis of social amoeba genomes highlights ancient originis for complex intercellular communication. Genome Res. 2011;21:1882-91.

34. Sucgang R, Kuo A, Tian X, Salerno W, Parikh A, Feasley CL, et al. Comparative genomics of the social amoebae Dictyostelium discoideum and Dictyostelium purpureum. Genome Biol. 2011;12:R20.

35. Goffeau A, Barrell BG, Bussey H, Davis RW, Dujon B, Feldmann H, et al. Life with 6000 genes. Science. 1996;274:546-67.

36. Kim JM, Vanguri S, Boeke JD, Gabriel A, Voytas DF. Transposable elements and genome organization: A comprehensive survey of retrotransposons revealed by the complete Saccharomyces cerevisiae genome sequence. Genome Res. 1998:8:464-78.

37. Hofmann J, Schumann G, Borschet G, Gosseringer R, Bach M, Bertling WM et al. Transfer RNA genes from Dictyostelium discoideum are frequently associated with repetitive elements and contain consensus boxes in their 5'-flanking and 3'-flanking regions. J Mol Biol. 1991:222:537-52.

38. Kaller M, Euteneuer U, Nellen W. Differential effects of heterochromatin protein 1 isoforms on mitotic chromosome distribution and growth in Dictyostelium discoideum. Eukaryot Cell. 2006;5:530-43.

39. Blus BJ, Wiggins K, Khorasanizadeh S. Epigenetic virtues of chromodomains. Crit Rev Biochem Mol Biol. 2011;46:507-26.

40. Boeke JD, Corces VG. Transcription and reverse transcription of retrotransposons. Ann Rev Microbiol. 1989;43:403-34.

41. Le Grice SFJ. "In the beginning": initiation of minus strand DNA synthesis in retroviruses and LTR-containing retrotransposons. Biochemistry. 2003;42: 14349-55.

42. Shadwick LL, Spiegel FW, Shadwick JD, Brown MW, Silberman JD. Eumycetozoa = Amoebozoa?: SSUrDNA phylogeny of protosteloid slime molds and its significance for the amoebozoan supergroup. PLoS One. 2009;: 4 :e6754.

43. Xie W, Gai X, Zhu Y, Zappulla DC, Sternglanz R, Voytas DF. Targeting of the yeast Ty5 retrotransposon to silent chromatin is medaited by interactions between integrase and Sir4p. Mol Cell Biol. 2001;21:6606-14.

44. Baller JA, Gao J, Stamenova R, Curcio MJ, Voytas DF. A nucleosomal surface defines an integration hotspot for the Saccharomyces cerevisiae Ty1 retrotransposon. Genome Res. 2012;22:704-13.

45. Mularoni L, Zhou Y, Bowen T, Gangadharan S, Wheelan SJ, Boeke JD. Retrotransposon Ty1 intgration targets specifically positioned asymmetric nucleosomal DNA segments in tRNA hotspots. Genome Res. 2012;22:693-703.

46. Bridier-Nahmias A, Tchalikian-Cosson A, Baller JA, Menouni R, Fayol H, Flores A, et al. An RNA polymerase III subunit determines sites of retrotransposon integration. Science. 2015;348:585-8.

47. Cheung S, Ma L, Chan PH, Hu HL, Mayor T, Chen HT, et al. Ty1-Integrase interacts with RNA Polymerase III specific subcomplexes to promote insertion of Ty1 elements upstream of Pol III-transcribed genes. J Biol Chem. 2016:291:6396-411.

48. Qi X, Daily K, Nguyen K, Wang HX, Mayhew D, Rigor P, et al. Retrotransposon profiling of RNA polymerase III initiation sites. Genome Res. 2012;22:681-92.

49. Siol O, Boutliliss M, Chung T, Glöckner G, Dingermann T, Winckler T. Role of RNA polymerase III transcription factors in the selection of integration sites by the Dictyostelium non-long terminal repeat retrotransposon TRE5-A. Mol Cell Biol. 2006;26:8242-51.

50. Levin HL. It's prime time for reverse transcriptase. Cell. 1997:88:5-8.

51. Levin HL. A novel mechanism of self-primed reverse transcription defines a new family of retroelements. Mol Cell Biol. 1995;15:3310-7.

52. Dean RA, Talbot NJ, Ebbole DJ, Farman ML, Mitchell TK, Orbach MJ, et al. The genome sequence of the rice blast fungus Magnaporthe grisea. Nature. 2005:434:980-6.

53. Dubin M, Fuchs J, Gräf R, Schubert I, Nellen W. Dynamics of a novel centromeric histone variant $\mathrm{CenH} 3$ reveals the evolutionary ancestral timing of centromere biogenesis. Nuc Acids Res. 2010;38:7526-37.

54. Novikov A, Smyshlyaev G, Novikova O. Evolutionary history of LTR retrotransposon chromodomains in plants. Int J Plant Genomics. 2012; 2012:874743.

55. Neumann P, Navrátilová A, Kobližzová A, Kejnovský E, Hřibová E, Hobza R, et al. Plant centromeric retrotransposons: a structural and cytogenetic perspective. Mob DNA. 2011;2:4. 
56. Basu S, Fey P, Pandit Y, Dodson R, Kibbe WA, Chisholm RL. dictyBase 2013 integrating multiple Dictyostelid species. Nuc Acids Res. 2013;41(Database issue):D676-D83.

57. Carver T, Bleasby A. The design of Jemboss: a graphical user interface to EMBOSS. Bioinformatics. 2003;19:1837-43.

58. Xu Z, Wang H. LTR_FINDER: an efficient tool for the prediction of full-length LTR retrotransposons. Nucleic Acids Res. 2007;35(Web Server issue):W265-W8.

59. Schattner P, Brooks AN, Lowe TM. The tRNAscan-SE, snoscan and snoGPS web servers for the detection of tRNAs and snoRNAs. Nucleic Acids Res. 2005;33(Web Server issue):W686-W9.

60. Laslett D, Canback B. ARAGORN, a program to detect tRNA genes and tmRNA genes in nucleotide sequences. Nucleic Acids Res. 2004;32:11-6.

61. Jurka J, Kapitonov W, Pavlicek A, Klonowski P, Kohany O, Walichiewicz J. Repbase Update, a database of eukaryotic repetitive elements. Cytogen Genome Res. 2005:110:462-7.

62. Marchler-Bauer A, Derbyshire MK, Gonzales NR, Lu S, Chitsaz F, Geer LY, et al. CDD: NCBI's conserved domain database. Nucleic Acids Res. 2015; 43(Database issue):D222-D6.

63. Thompson JD, Gibson TJ, Plewniak F, Jeanmougin F, Higgins DG. The ClustalX windows interface: flexible strategies for multiple sequence alignment aided by quality analysis tools. Nuc Acids Res. 1997;24:4876-82.

64. Kumar S, Stecher G, Tamura K. MEGA7: Molecular Evolutionary Genetics Analysis version 7.0 for bigger datasets. Mol Biol Evol. 2016;Mar 22. pii: msw054. [Epub ahead of print].

65. Saitou N, Nei M. The neighbor-joining method: A new method for reconstructing phylogenetic trees. Mol Biol Evol. 1987;4:406-25.

66. Jones DT, Taylor WR, Thornton JM. The rapid generation of mutation data matrices from protein sequences. Comput Appl Biosci. 1992;8:275-82.

67. Marschalek R, Hofmann J, Schumann G, Gosseringer R, Dingermann T. Structure of DRE, a retrotransposable element which integrates with position specificity upstream of Dictyostelium discoideum tRNA genes. Mol Cell Biol. 1992;12:229-39.

68. Felsenstein J. Confidence limits on phylogenies: An approach using the bootstrap. Evolution. 1985;39:783-91.

69. Male G, von Appen A, Glatt S, Taylor NM, Cristovao M, Groetsch H, et al. Architecture of TFIIIC and its role in RNA polymerase III pre-initiation complex assembly. Nat Commun. 2015;6:7387.

\section{Submit your next manuscript to BioMed Central and we will help you at every step:}

- We accept pre-submission inquiries

- Our selector tool helps you to find the most relevant journal

- We provide round the clock customer support

- Convenient online submission

- Thorough peer review

- Inclusion in PubMed and all major indexing services

- Maximum visibility for your research

Submit your manuscript at www.biomedcentral.com/submit 